\title{
Choice of management strategy for colorectal cancer based on a diagnostic immunohistochemical test for defective mismatch repair
}

L Cawkwell, S Gray, H Murgatroyd, F Sutherland, L Haine, M Longfellow, S O'Loughlin, D Cross, O Kronborg, C Fenger, N Mapstone, M Dixon, P Quirke

\begin{abstract}
Background-Despite intensive research into the molecular abnormalities associated with colorectal cancer (CRC), no diagnostic tests have emerged which usefully complement standard histopathological assessments.
\end{abstract}

Aims-To assess the feasibility of using immunohistochemistry to detect replication error (RER) positive CRCs and determine the incidence of RER positivity within distinct patient subgroups.

Methods-502 CRCs were analysed for RER positivity (at least two markers affected) and/or expression of hMSH2 and hMLH1.

Results-There were $15 / 30$ (50\%) patients with metachronous CRCs, 16/51 (31\%) with synchronous CRCs, 14/45 (31\%) with a proximal colon carcinoma, and 4/23 (17\%) who developed a CRC under the age of 50 showed RER positivity. However, 0/54 patients who developed a solitary carcinoma of the rectum/left colon over the age of 50 showed RER positivity. Immunohistochemical analysis revealed that $66 / 66$ $(100 \%)$ RER positive carcinomas were associated with complete lack of expression of either hMSH2 or hMLH1. This correlation was confirmed using a further 101 proximal colon carcinomas. Patients with a mismatch repair defective carcinoma showed improved survival but a 5.54 times relative risk of developing a metachronous CRC. A prospective immunohistochemical study revealed 13/117 (11\%) patients had a mismatch repair defective carcinoma. A fivefold excess of hMLH1 defective cases was noted.

Conclusions-All RER positive carcinomas were identified by the immunohistochemical test. This is the first simple laboratory test which can be performed routinely on all CRCs. It will provide a method for selecting patients who should be investigated for HNPCC, offered long term follow up, and who may not respond to standard chemotherapy regimens. (Gut 1999;45:409-415)

Keywords: colorectal cancer; mismatch repair; hMSH2; hMLH1; microsatellite instability; immunohistochemistry

Dr L Cawkwell, Molecular Oncology, Algernon Firth Institute of Pathology, Schoo of Medicine, University of Leeds, Leeds LS2 9JT, UK

Accepted for publication 3 March 1999

Colorectal cancer is the second most common cause of death from cancer in the UK with approximately 20000 people per year dying from the disease. It is an excellent model for multistep carcinogenic progression and much research has been directed at unravelling the molecular pathways which lead to a colorectal carcinoma. However, this molecular and genetic research has, as yet, not resulted in the introduction of any new laboratory tests which would be of assistance in the choice of management strategy, beyond that given by the current standard histopathological assessments of tumour stage and grade.

Hereditary non-polyposis colorectal cancer (HNPCC) is an autosomal dominant condition which accounts for $2-5 \%$ of colorectal cancer cases. ${ }^{1}$ HNPCC carcinomas tend to develop at an early age, are usually found on the right side of the colon, and are less aggressive than sporadic colorectal cancers. ${ }^{12}$ In addition, synchronous and metachronous colorectal cancers occur more frequently in patients with HNPCC, ${ }^{3}$ with around $40 \%$ of patients developing a further carcinoma within 10 years if a total colonic resection is not performed as the definitive operation. ${ }^{4}$ HNPCC is caused by a defect in the DNA mismatch repair pathway, with the majority of cases having germline mutations in the hMSH2 or hMLH1 genes. ${ }^{5}$ Such mutations often lead to the production of a truncated protein. ${ }^{5}$ In somatic tissue the second functional copy of the gene may then be inactivated either by allele loss, hypermethylation of the promoter region to silence transcription, or further mutation. Errors at replication are corrected via the DNA mismatch repair pathway and any defect within this system will lead to the rapid accrual of genome wide mutations.

Tumours which have defective mismatch repair show a characteristic molecular phenotype, termed microsatellite instability, ${ }^{6}$ as repeated DNA sequences (microsatellites) are often replicated incorrectly. If the mismatch repair system does not correct these errors, these regions of DNA can become extended or shortened in relation to the original template. HNPCC tumours characteristically show a high frequency of altered microsatellite sequences (over $29 \%$ of microsatellites tested would be affected) and are also termed replication error (RER) positive. Approximately 10\%

Abbreviations used in this paper: $\mathrm{CRC}$, colorectal cancer; FAP, familial adenomatous polyposis; HNPCC, hereditary non-polyposis colorectal cancer; PCR, polymerase chain reaction; RER, replication 
of sporadic colorectal cancers are also RER positive. ${ }^{78}$ Importantly, as these carcinomas often show characteristics in common with HNPCC tumours, these patients should be investigated to rule out this inherited condition. Patients with a sporadic RER positive colorectal carcinoma seem to have a better prognosis than patients whose carcinoma is RER negative, ${ }^{7-9}$ which has implications for clinical management of these patients. These patients are also at risk of metachronous colorectal cancers ${ }^{10}$ and therefore require long term follow up. There is also accumulating evidence to suggest that RER positive tumours respond differently to chemotherapy. ${ }^{11}{ }^{12}$ This issue needs to be addressed urgently to avoid unnecessary administration of expensive and unpleasant chemotherapeutic drugs to patients who will not benefit. These factors are sufficient to suggest that sporadic RER positive colorectal carcinomas constitute a distinct subgroup of colorectal cancers and as such should be identified and appropriately managed. In order to identify these RER positive carcinomas a microsatellite instability test based on the polymerase chain reaction (PCR) can be used; however, this requires the extraction of DNA and specialised equipment, is time consuming, and is not ideally suited to all histopathology laboratories. Due to the time and cost involved it is not a feasible option to test routinely all colorectal carcinomas using this approach. It would therefore be beneficial to identify subgroups of patients where most RER positive cases are likely to be found. Ideally, however, the identification of a simple, routinely applicable test for detecting RER positivity, which could be applied to all colorectal carcinomas, is required.

The aim of this study was to assess the frequency of RER positive carcinomas within easily identifiable phenotypic subgroups of sporadic colorectal cancer patients. This would allow the identification of any groups of patients who should be targeted for a routine PCR based microsatellite instability test due to their increased risk of harbouring an RER positive carcinoma. Secondly, the status of hMSH2 and hMLH1 would be assessed using an immunohistochemical approach in order to indicate the frequency with which RER positive cases could be detected using this simple approach alone.

\section{Materials and methods}

PATIENT SUBGROUPS

In order to investigate the frequency of RER positive colorectal carcinomas in a variety of easily definable patient subgroups the following series were assessed.

Early onset group - This consisted of 23 nonfamilial adenomatous polyposis (non-FAP) patients from the General Infirmary at Leeds who developed colorectal cancer under the age of 50 . Within this group there were 13 patients who were under 40 years at diagnosis.

Synchronous colorectal cancer group-This consisted of 51 patients from the General Infirmary at Leeds who presented with at least two synchronous colorectal carcinomas. Where possible, all carcinomas were investigated (a total of 106).

Metachronous colorectal cancer group-In order to identify patients who develop a second primary colorectal cancer, a prospective follow up study is required; 21 such patients were therefore identified from two prospective studies involving 900 colorectal cancer patients at Odense University Hospital in Denmark. Where possible, all carcinomas were examined (a total of 47). A further series of nine patients from the General Infirmary at Leeds who had developed more than one primary colorectal cancer metachronously were identified. In all cases only the most recent carcinoma was available for assay.

Late onset group - Sporadic colorectal cancers usually occur in patients over the age of 50 years and 99 such patients from the General Infirmary at Leeds were identified. These were then separated according to carcinoma location into left and right colon, in view of the association between RER positivity and right sided colorectal carcinomas. There were 54 left sided carcinomas (from the rectum, sigmoid, and descending colon) and 45 right sided carcinomas (from the caecum, ascending colon, and transverse colon).

\section{PCR BASED MICROSATELLITE INSTABILITY ASSAY}

Following histological assessment and microdissection, DNA was extracted from formalin fixed, paraffin wax embedded carcinoma and normal tissue for each patient using a rapid method developed for use with the Nucleon DNA extraction kit (Scotlab Ltd, Strathclyde). ${ }^{13}$ The fluorescent multiplex microsatellite instability assay, as previously described, ${ }^{14}$ was used with a panel of five markers: D13S175, ${ }^{6}$ BAT-25, ${ }^{15}$ MycL $1,{ }^{16}$ D5S346, ${ }^{17}$ and p53(2). ${ }^{18}$ This is a limited version of the diagnostic microsatellite instability assay in use in this department. ${ }^{19}$ If at least two markers showed microsatellite instability (fig 1) the carcinoma was classified as RER positive.

IMMUNOHISTOCHEMICAL STAINING OF hMSH2 AND hMLH1

One block of formalin fixed, paraffin wax embedded adenocarcinoma tissue was selected per case. Wherever possible, this block included a region of normal mucosa adjacent to the carcinoma. Three $4 \mu \mathrm{m}$ sections per case were cut onto Superfrost Plus microscope slides (Merck $\mathrm{Ltd}$, Poole, UK) and allowed to dry at $37^{\circ} \mathrm{C}$ overnight. Immunohistochemical analysis of both antibodies was performed in parallel using an identical method and therefore one slide served as a negative control (antibody omitted) for both reactions. Following dewaxing and rehydration the endogenous peroxidase was blocked using $2 \%$ hydrogen peroxide in methanol for 20 minutes. Antigenic site retrieval was accomplished by boiling under full pressure (103 Kpa) for 90 seconds in 1.5 litres of $1 / 100$ Antigen Unmasking Solution (Vector Laboratories Ltd, Peterborough, UK) in a stainless steel pressure cooker (Prestige). Immunohisto- 


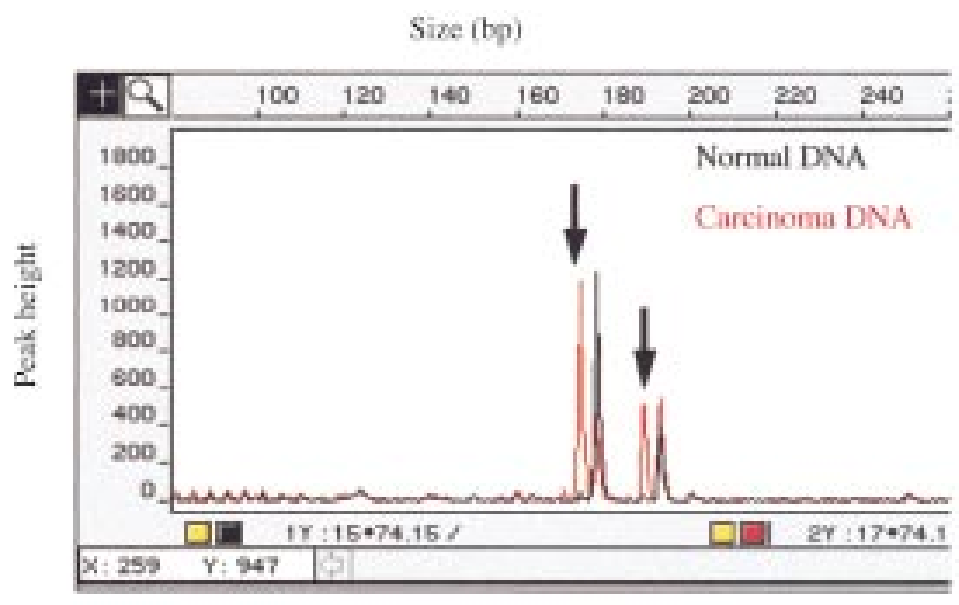

Figure 1 Detection of microsatellite instability using the MycL1 marker and the fluorescent PCR based assay. On the cross sectional representation the alleles from the carcinoma DNA and the corresponding normal DNA have been superimposed. The constitutional alleles, which are common to both DNA samples, are colocalised and the novel alleles in the carcinoma DNA (arrowed) are clearly evident.

chemical reactions were performed using the Sequenza system (Shandon, Basingstoke, UK). Non-specific binding was prevented by incubation for five minutes in a solution of $1 \times$ casein (Vector Laboratories Ltd) in Tris buffered saline ( $\mathrm{pH}$ 7.6). Endogenous biotin was blocked in accordance with the instructions of the Avidin/Biotin Blocking Kit (Vector Laboratories Ltd). The antibodies were then applied for two hours at room temperature. The monoclonal hMSH2 antibody (catalogue number NA27, Calbiochem, Nottingham, UK) was used at a final dilution of $1 / 100$ made up in a solution of $0.2 \times$ casein in Tris buffered saline $(\mathrm{pH}$ 7.6). The monoclonal hMLH1 antibody (catalogue number 13291A, Pharmingen, San Diego, California, USA) was used at a final dilution of $1 / 70$ made up in a solution of $0.2 \times$ casein in Tris buffered saline $(\mathrm{pH}$ 7.6). A StreptABComplex/HRP Duet Kit (catalogue number K0492, Dako Ltd, High Wycombe, UK) was applied in accordance with the manufacturer's instructions. The reactions were visualised using $0.02 \%$ diaminobenzidine in Tris buffered saline $(\mathrm{pH}$ 7.6). The contrast was enhanced using a solution of $0.5 \%$ copper sulphate in $0.9 \%$ saline. The sections were counterstained with haematoxylin. All results were scored without knowledge of the microsatellite instability results. Loss of expression was recorded when nuclear staining was seen in normal tissue but not in adjacent malignant cells.

CONFIRMATION OF IMMUNOHISTOCHEMICAL DETECTION OF RER POSITIVITY

A further series of 101 patients from the General Infirmary at Leeds who had developed a solitary carcinoma on the right side of the colon over the age of 50 was identified. Using one block per carcinoma, the immunohistochemical method for hMSH2 and hMLH1 was initially performed. All carcinomas which showed lack of expression with either antibody, as well as six carcinomas which showed no loss of expression, were then investigated using the fluorescent microsatellite instability assay. RER positivity (at least two markers affected) was scored without knowledge of the immunohistochemical status of hMSH2 and hMLH1. These microsatellite instability assays were carried out without the need for DNA extraction by using a method for direct PCR amplification from small microdissected areas of tissue as described previously. ${ }^{20}$

\section{SURVIVAL ANALYSIS}

The 101 right sided colonic carcinomas which were used to confirm that RER positivity could be detected by immunohistochemistry alone were also used to assess patient survival according to the mismatch repair status of the carcinoma. Two patients could not be traced but 99/101 were followed up for five years from the time of operation. Kaplan-Meier survival analyses were carried out using SPSS for Windows, with an event defined as death from colon cancer. The log rank test was used to assess the statistical significance of the survival data.

PROSPECTIVE FEASIBILITY STUDY

To assess the feasibility of introducing the immunohistochemical test for hMLH1 and $\mathrm{hMSH} 2$ for routine histopathological diagnosis we prospectively analysed all cases of colorectal cancer diagnosed in the Histopathology Department of the General Infirmary at Leeds between 1 January and 18 September 1998. This series consisted of 117 patients.

\section{Results}

FREQUENCY OF REPLICATION ERROR POSITIVITY IN PATIENT SUBGROUPS

The patient subgroups investigated in this study were readily identifiable according to the characteristics used such as patient age, multiplicity of colorectal carcinomas, or location of the carcinoma. The RER positivity among the subgroups used (table 1) indicates that the highest frequencies are seen in the patients with an early onset colorectal carcinoma, or with multiple carcinomas (synchronous or metachronous), or with a carcinoma on the right side of the colon. None of the 54 left sided, later onset carcinomas showed RER positivity.

PREDICTION OF METACHRONOUS COLORECTAL CARCINOMA RISK

The subgroups of patients used in the study who had developed more than one primary colorectal carcinoma metachronously revealed a high frequency of RER positivity (table 1). However, such patients would need to develop a second carcinoma before they could be placed into this subgroup and may therefore not be immediately targeted for routine RER testing. We assessed the possible predictive value of RER positivity using the prospective series of metachronous colorectal cancers which developed in a cohort of 900 patients from Denmark, where tissue from the first primary carcinoma in each case was available. A total of $8 / 21(38 \%)$ of these index colorectal carcinomas was RER positive. Assuming a background RER positivity rate of $10 \%,{ }^{78}$ the 

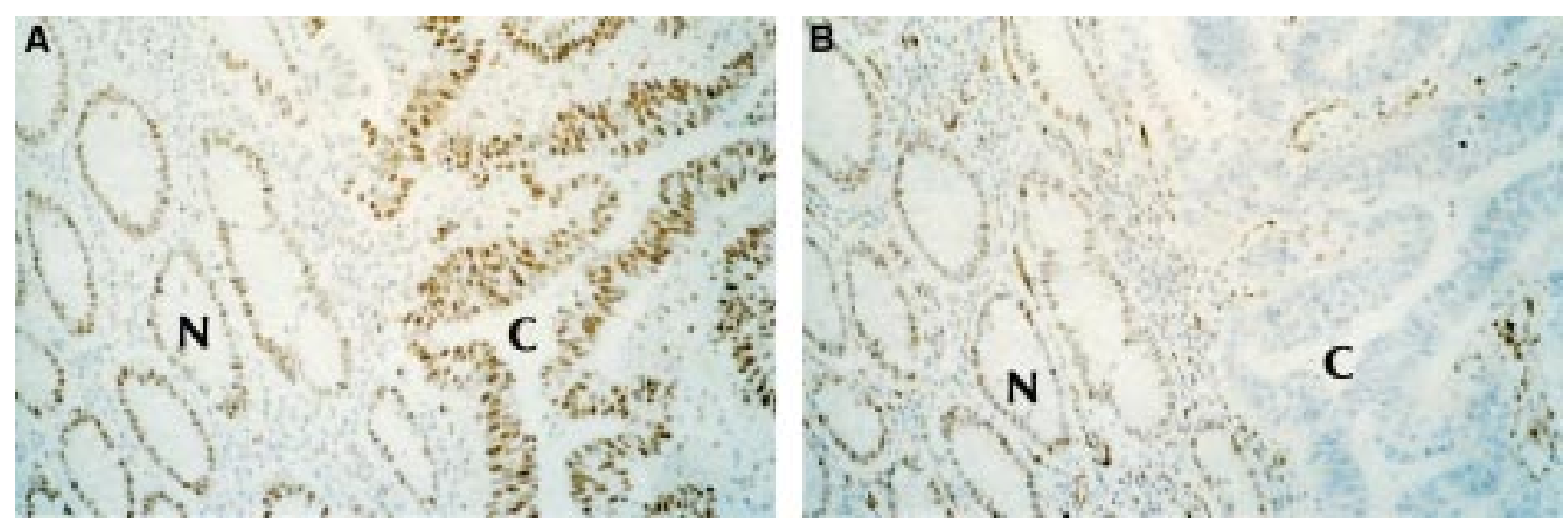

Figure 2 Immunohistochemical staining of $h M S H 2$ and hMLH1 using consecutive tissue sections (original magnification $\times 160$ ). (A) Positive staining of normal control mucosa $(N)$ and adjacent carcinoma $(C)$ with hMSH2. (B) Positive staining of normal control mucosa $(N)$; loss of $h M L H 1$ protein expression in the adjacent carcinoma $(C)$ is clearly evident. Intervening stromal cells are positive.

relative risk of a patient with an RER positive first cancer subsequently developing a metachronous colorectal cancer is 5.54 (95\% confidence interval 2.36-13.0) times that of a patient whose carcinoma is not RER positive.

IMMUNOHISTOCHEMICAL DETECTION OF RER POSITIVE CARCINOMAS

The nuclei of the cells occupying the lower two thirds of normal colonic crypts stain positively with both antibodies and wherever possible a block which contains both carcinoma and normal mucosa, to serve as an internal positive control, should be chosen. The scoring of both antibodies was straightforward and clear cut as no carcinomas showed heterogeneity of staining, with tumour cells in all cases being either totally positive or totally negative (fig 2 ). No carcinoma showed loss of staining with both hMSH2 and hMLH1. Table 1 presents the results. All RER positive cases were identified with the use of these two antibodies.
INTERTUMOUR VARIATION IN RER POSITIVITY

For the patients with synchronous colorectal cancers and the Danish patients with metachronous cancers, more than one carcinoma per patient was assayed. Several of these patients had developed both RER positive and RER negative carcinomas. Both the PCR based microsatellite instability assay and the immunohistochemical assessment revealed the same findings. Of the 16 patients in the synchronous subgroup who had at least one carcinoma which was RER positive, five had developed a synchronous carcinoma which was RER negative. In the Danish metachronous colorectal cancer subgroup there were 12 patients who had at least one RER positive carcinoma. Four of these patients also exhibited carcinomas which were RER negative. The carcinomas from the other eight patients were all RER positive but, interestingly, two of these patients showed a combination of hMSH2 and hMLH1 defective cancers.

Table 1 Assessment of colorectal cancer patient subgroups: frequency of RER positivity detected using a PCR based microsatellite instability assay and hMSH2/hMLH1 immunohistochemical findings for RER positive carcinomas

\begin{tabular}{|c|c|c|c|c|c|c|c|}
\hline \multirow[b]{2}{*}{ Colorectal cancer patient subgroup } & \multirow[b]{2}{*}{$\begin{array}{l}\text { Number of } \\
\text { patients }\end{array}$} & \multirow[b]{2}{*}{$\begin{array}{l}\text { Number of patients } \\
\text { with an RER } \\
\text { positive carcinoma } \\
\text { (PCR based assay) }\end{array}$} & \multirow[b]{2}{*}{$\begin{array}{l}\text { Number of } \\
\text { individual } \\
\text { carcinomas } \\
\text { investigated }\end{array}$} & \multicolumn{3}{|c|}{$\begin{array}{l}\text { Immunohistochemistry results } \\
\text { (RER positive carcinomas) }\end{array}$} & \multirow[b]{2}{*}{$\begin{array}{l}\text { Concordance of RER } \\
\text { positivity and loss of } \\
h M S H 2 / h M L H 1^{*}\end{array}$} \\
\hline & & & & $\begin{array}{l}\text { Loss of } \\
\text { hMSH2 } \\
\text { staining }\end{array}$ & $\begin{array}{l}\text { Loss of } \\
\text { hMLH1 } \\
\text { staining }\end{array}$ & $\begin{array}{l}\text { Tissue not } \\
\text { available }\end{array}$ & \\
\hline \multicolumn{8}{|l|}{ Age at operation: over $50 \mathrm{y}$} \\
\hline \multicolumn{8}{|l|}{ Carcinoma site: rectum or left colon } \\
\hline \multicolumn{8}{|l|}{ No synchronous CRCs } \\
\hline No known previous CRC & 54 & $0 / 54$ & 54 & 0 & 0 & 0 & - \\
\hline \multicolumn{8}{|l|}{ Age at operation: over $50 \mathrm{y}$} \\
\hline \multicolumn{8}{|l|}{ Carcinoma site: right colon } \\
\hline \multicolumn{8}{|l|}{ No synchronous CRCs } \\
\hline No known previous CRC & 45 & $14 / 45(31 \%)$ & 45 & 1 & 13 & 0 & $100 \%(14 / 14)$ \\
\hline \multicolumn{8}{|l|}{ Early onset } \\
\hline \multicolumn{8}{|l|}{ Age at operation: } \\
\hline under $50 \mathrm{y}$ & 23 & $4 / 23(17 \%)$ & 23 & 1 & 2 & 1 & $100 \%(3 / 3)$ \\
\hline Synchronous CRC & 51 & $16 / 51(31 \%)$ & 106 & 7 & 16 & 7 & $100 \%(23 / 23)$ \\
\hline \multicolumn{8}{|l|}{ Metachronous CRC } \\
\hline Leeds & 9 & $3 / 9(33 \%)$ & 9 & 1 & 1 & 1 & $100 \%(2 / 2)$ \\
\hline Denmark & 21 & $12 / 21(57 \%)$ & 47 & 3 & 21 & 0 & $100 \%(24 / 24)$ \\
\hline \multicolumn{8}{|l|}{ Confirmatory series } \\
\hline Right sided sporadic CRCs & 101 & - & 101 & 1 & 15 & 0 & $100 \%(16 / 16)$ \\
\hline \multicolumn{8}{|l|}{ Prospective series } \\
\hline All CRCs diagnosed 1 Jan to 18 Sept 1998 & 117 & - & 117 & 2 & 11 & 0 & - \\
\hline Totals & 421 & - & 502 & 16 & 79 & 9 & $100 \%(82 / 82)$ \\
\hline
\end{tabular}

CRC, colorectal carcinoma.

^For cases tested. 


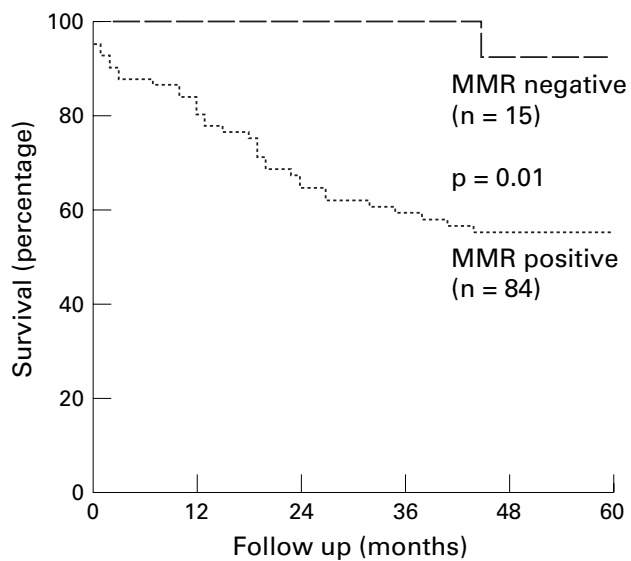

Figure 3 Kaplan-Meier survival curve for mismatch repair status, as defined by the expression of hMSH2 and $h M L H 1$, for proximally located sporadic colon carcinomas. A significantly increased patient survival $(p=0.01)$ was associated with the 15 mismatch repair defective (MMR negative) cases (14 lacking $h M L H 1$, one lacking $h M S H 2$ ) versus the 84 mismatch repair proficient (MMR positive) cases.

LOW FREQUENCY MICROSATELLITE INSTABILITY In the PCR based microsatellite instability study, 14 carcinomas were identified which exhibited instability at only one microsatellite marker. This was not a sufficiently high frequency for the carcinoma to be classified as RER positive. The immunohistochemical analysis of $\mathrm{hMSH} 2$ and $\mathrm{hMLH} 1$ in these cases revealed that they were not associated with loss of expression of either of these two DNA repair proteins.

CONFIRMATION OF IMMUNOHISTOCHEMICAL DETECTION OF RER POSITIVITY

In order to confirm that the loss of expression of either hMSH2 or hMLH1 detected by immunohistochemistry was always associated with the RER positive phenotype, a further series of 101 sporadic right sided colon carcinomas was assessed. A total of 16/101 $(15.8 \%)$ carcinomas showed loss of protein expression of either hMSH2 or hMLH1. A preponderance of hMLH1 abnormalities was again noted, with 15 of these cases being defective in hMLH1. All 16 cases which showed a loss of expression were then subjected to the microsatellite instability assay and 16/16 cases were classified as RER positive. In six control cases, which showed no loss of protein expression, no microsatellite instability was detected. A significant $(p=0.01)$ survival advantage was associated with the patients who had a mismatch repair defective carcinoma, defined by loss of expression of either hMSH2 or hMLH1 (fig 3).

PROSPECTIVE FEASIBILITY STUDY

Successful hMSH2 and hMLH1 immunohistochemistry results were obtained for all 117 cases of colorectal cancer diagnosed in the Histopathology Department of the General Infirmary at Leeds between 1 January and 18 September 1998. This prospective study revealed a total of $13(11 \%)$ mismatch repair defective carcinomas (table 1) and a preponderance $(5: 1)$ of hMLH1 defective cases was again noted. Interestingly, 12 of these 13 patients had multiple primary colorectal carcinomas or a solitary proximal colon carcinoma.

\section{Discussion}

Although intensive research into the molecular abnormalities associated with sporadic colorectal cancer has been in progress for many years, as yet, no new clinically valuable routine tests to complement staging and grading have been introduced.

It is now clear that there is a distinct subset of sporadic colorectal cancers, which are distinguished by RER positivity. Patients who harbour such carcinomas may be carrying a germline HNPCC mutation. They seem to have an improved prognosis, but are at higher risk of further colorectal cancers. They may also be unnecessarily subjected to adjuvant chemotherapy to which they may not respond or may not require due to the good prognosis associated with RER positive carcinomas. These patients need to be identified and offered more appropriate management.

It is not feasible for all histopathology departments to screen all colorectal carcinomas routinely for RER positivity using a PCR based microsatellite instability assay. In this paper we have highlighted certain subgroups of patients, with a readily identifiable phenotype, who are most likely to harbour an RER positive carcinoma. It seems that patients who are over the age of 50 when they present with a first and solitary carcinoma which is located on the left side of the colon would be of lowest priority for such a test. As approximately two thirds of the total of solitary sporadic colorectal carcinomas develop on the left side of the colon, this would significantly decrease the workload. However, RER positive left sided carcinomas could be missed if this strategy was used. Indeed, in this study, 2/8 of the RER positive first carcinomas from the Danish patients who developed a second primary were located on the left side of the colon. It has also been reported that RER positive distal colorectal carcinomas are frequently associated with a significant family history of the disease. ${ }^{7}$ There are further drawbacks to the use of a PCR based microsatellite instability test for the detection of RER positive carcinomas. The assay is relatively complex, expensive, and takes at least 24 hours to complete. It will therefore not be feasible for histopathology departments to use this as a routine diagnostic assay on all cases. However, we believe that all colorectal cancers should be screened for RER positivity, especially if the apparent predictive value associated with metachronous cancer development is confirmed. The optimised immunohistochemical method for hMSH2 and hMLH1 given in this manuscript is robust and rapid (from sectioning to scoring takes 24 hours). Analysing a total of 502 carcinomas, we have confirmed the association of RER positivity and loss of hMSH2/hMLH1 protein expression reported in smaller studies. ${ }^{21}{ }^{22}$ All RER positive cases were identified with the use of these two antibodies, which indicates that the use of antibodies to the minor mismatch repair enzymes (PMS1, PMS2, hMSH6/ 
GTBP, hMSH3) would not be required. It would seem that all RER positive carcinomas have a complete lack of one of the major mismatch repair proteins. Carcinomas showing only low frequency microsatellite instability (one marker affected) were not associated with loss of expression of either hMSH2 or hMLH1, a finding also reported by Thibodeau et al. ${ }^{23}$ As was recently reported, ${ }^{23}$ we also noted an apparent preponderance (overall 5:1) of hMLH1 abnormalities over hMSH2 defects and all eight of the RER positive first carcinomas from the metachronous colorectal cancer patients in the Danish prospective series were associated with a defective hMLH1. It has recently been reported that loss of hMLH1 expression correlates with hypermethylation of its promoter, ${ }^{24}{ }^{25}$ and it could be hypothesised that an excess of hMLH1 abnormalities could be produced if the hMSH2 promoter is less likely to be inactivated via this mechanism. Loss of expression of hMSH2 or hMLH1 was not associated with any case which did not show RER positivity. We have also established that the survival advantage associated with RER positive proximal colon carcinomas ${ }^{9}$ is also evident from the analysis of expression of the hMSH2 and hMLH1 proteins. It would seem that the survival advantage for RER positive sporadic colorectal cancers ${ }^{7-9}$ may be accounted for by the proximally located subgroup, as from this study it seems that mismatch repair deficiency/RER positivity is very infrequent among left sided cancers.

The immunohistochemical test for hMSH2 and hMLH1 yielded both prognostic information and a prediction of metachronous colorectal cancer risk. We therefore assessed the feasibility of introducing the immunohistochemical test for hMLH1 and hMSH2 for routine histopathological diagnosis in our department by prospectively analysing all new cases of colorectal cancer between 1 January and 18 September 1998. We obtained successful hMSH2 and hMLH1 immunohistochemistry results for all 117 cases and identified $13(11 \%)$ mismatch repair defective carcinomas (table 1). A preponderance $(5: 1)$ of hMLH1 defective cases was again noted. The frequency of mismatch repair defective carcinomas in this prospective group $(13 / 117 ; 11 \%)$ is in close agreement with the RER positivity incidence for sporadic colorectal cancers. ${ }^{78}$ Interestingly, 12 of the 13 patients, who had a mismatch repair defective carcinoma, had multiple primary colorectal carcinomas or a solitary proximal colon carcinoma. This finding further confirms the data presented here regarding which patient subgroups show the highest incidence of mismatch repair deficiencies.

This simple laboratory test will now allow histopathology departments to screen all colorectal carcinomas routinely for RER positivity in the same way that breast cancers are screened for oestrogen receptor expression. The intertumour variation seen in patients with multiple colorectal carcinomas indicates that all carcinomas from such cases should be examined if RER positive cases are not to be overlooked.
In vitro data suggest that RER positive tumours may be resistant to certain types of chemotherapy. ${ }^{11}{ }^{12}$ We urgently require in vivo data in order to predict how patients with RER positive carcinomas respond to chemotherapy. When the results are known we may also need to offer specific adjuvant therapy to patients with a carcinoma which is found to be RER positive following routine testing.

Patients who are found to have an RER positive carcinoma by immunohistochemical analysis should be investigated for HNPCC, initially through assessing their family history of cancer, but also, after genetic counselling, by mutation screening. The immunohistochemical assay should reduce the time and costs of DNA sequencing by indicating which gene to analyse for the presence of a germline mutation.

We would also suggest that patients who are found to have an RER negative carcinoma (no loss of hMSH2 or hMLH1 protein expression) could be released from hospital follow up after three years. This is sufficient time for most disease recurrences to have been detected. Only patients with RER positive carcinomas or other high risk factors, who are at increased risk of further colorectal primaries, should be surveyed colonoscopically for at least 10 years. This would lead to a substantial reduction in workload and the financial costs of endoscopy. Only a small proportion of patients who have an RER negative carcinoma but develop a metachronous carcinoma after the three year follow up period would possibly be disadvantaged by the adoption of this management strategy.

Our findings show that immunohistochemical staining of hMSH2 and hMLH1 alone will detect all RER positive sporadic colorectal cancers. We believe that this is the first simple immunohistochemical test which will provide valuable management information in addition to histopathological assessment of tumour stage and grade and which can be performed routinely on all sporadic colorectal carcinomas. With more clinical research into chemotherapeutic responses of patients who have an RER positive carcinoma the value of this routine test may increase even further.

This work was supported by Yorkshire Cancer Research and the Danish Cancer Society. We are grateful to Mr Fraser Lewis for oligonucleotide synthesis, Mr Dawood Dassu of the Yorkshire Clinical Trials and Research Unit for statistical analysis, and the Northern and Yorkshire Cancer Registry and Information Service for providing data on patient outcomes.

\footnotetext{
1 Lynch HT, Smyrk T, Lynch J. An update of HNPCC (Lynch syndrome). Cancer Genet Cytogenet 1997;93:84-99.

2 Sankila R, Aaltonen LA, Jarvinen HJ, et al. Better survival rates in patients with hMLH1-associated hereditary colorectal cancer. Gastroenterology 1996;110:682-7.

3 Fante R, Roncucci L, Di Gregorio C, et al. Frequency and clinical features of multiple tumors of the large bowel in the general population and in patients with hereditary colorectal carcinoma. Cancer 1996;77:2013-21.

4 Lynch HT, Smyrk T, Lynch JF. Overview of natural history, pathology, molecular genetics and management of 5 Liu B, Parsons R, Papadopoulos N, et al. Analysis of mismatch repair genes in hereditary non-polyposis colorectal cancer patients. Nat Med 1996;2:169-74.

6 Aaltonen LA, Peltomaki P, Leach FS, et al. Clues to the pathogenesis of familial colorectal cancer. Science 1993; 260:812-16.

7 Lothe RA, Peltomaki P, Meling GI, et al. Genomic instability in colorectal cancer: relationship to clinicopathological variables and family history. Cancer Res 1993;53:5849-52.
} 
8 Bubb VJ, Curtis LJ, Cunningham C, et al. Microsatellite instability and the role of hMSH2 in sporadic colorectal instability and the role of hMSH
cancer. Oncogene 1996;12:2641-9.

9 Thibodeau SN, Bren G, Schaid D. Microsatellite instability in cancer of the proximal colon. Science 1993;260:816-19.

10 Sengupta SB, Yiu CY, Boulos PB, et al. Genetic instability in patients with metachronous colorectal cancers. Br F Surg 1997;84:996-1000.

11 Branch P, Hampson R, Karran P. DNA mismatch binding defects, DNA damage tolerance, and mutator phenotypes in human colorectal carcinoma cell lines. Cancer Res 1995; 55:2304-9.

12 Boland CR. Roles of the DNA mismatch repair genes in colorectal tumorigenesis. Int f Cancer 1996;69:47-9.

13 Cawkwell L, Quirke P. Comparison and development of DNA extraction methods for formalin-fixed, paraffinembedded tissue [abstract]. F Pathol 1997;182(suppl):22A

14 Cawkwell L, Li D, Lewis FA, et al. Microsatellite instability in colorectal cancer: improved assessment using fluoresin colorectal cancer: improved assessment using fluorescent poly $465-71$.

15 Liu B, Farrington SM, Petersen GM, et al. Genetic instability occurs in the majority of young patients with colorectal

16 Makela TP, Hellsten E, Vesa J, et al. An Alu variable polyA repeat polymorphism upstream of L-myc at $1 \mathrm{p} 32$. Hum Mol Genet 1992;1:217.

17 Spirio L, Nelson L, Ward K, et al. A CA-repeat polymorphism close to the adenomatous poyposis col (APC) gene offers improved diagnostic testing for familia APC. Am f Hum Genet 1993;52:286-96.
18 Cawkwell L, Lewis FA, Quirke P. Frequency of allele loss of DCC, p53, RB1, WT1, NF1, NM23 \& APC/MCC in colorectal cancer assayed by fluorescent multiplex polymerase chain reaction. Br f Cancer 1994;70:813-18. Cawkwell L, Kennedy A, Lewis F, et al. Optimisation of a
fluorescent assay for allelic imbalance and microsatellite instability [abstract]. F Pathol 1997;182(suppl):22A

20 Cawkwell L, Quirke P. Direct multiplex amplification of formalin-fixed, paraffin-embedded tissue biopsies [abstract]. F Pathol 1997;182(suppl):22A

21 Thibodeau SN, French AJ, Roche PC, et al. Altered expression of hMSH2 and hMLH1 in tumors with microsatellite instability and genetic alterations in mismatch repair genes. Cancer Res 1996;56:4836-40.

22 Dietmaier W, Wallinger S, Bocker $\mathrm{T}$, et al. Diagnostic microsatellite instability: definition and correlation with mismatch repair protein expression. Cancer Res 1997;57: mismatch

23 Thibodeau SN, French AJ, Cunningham JM, et al. Microsatellite instability in colorectal cancer: different mutator phenotypes and the principal involvement of hMLH1. phenotypes and the princip
Cancer Res 1998;58:1713-18.

24 Kane MF, Loda M, Gaida GM, et al. Methylation of the hMLH1 promoter correlates with lack of expression of hMLH1 in sporadic colon tumors and mismatch repairdefective human tumor cell lines. Cancer Res 1997;57:80811

25 Herman JG, Umar A, Polyak K, et al. Incidence and unctional consequences of hMLH1 promoter hypermethylation in colorectal carcinoma. Proc Natl Acad Sci USA 1998;95:6870-5. 